\title{
The future of pediatric hepatocellular carcinoma: a combination of surgical, locoregional, and targeted therapy
}

\author{
Víola B. Weeda' ${ }^{1}$ Maciej Murawski \\ 'Department of Surgery, Academic Medical Centre, University of Amsterdam, Amsterdam 1105AZ, the Netherlands. \\ ${ }^{2}$ Department of Pediatric Surgery and Urology, Medical University of Gdansk, Gdansk 80-210, Poland.
}

Correspondence to: Dr. Víola B. Weeda, Department of Surgery, Academic Medical Centre, University of Amsterdam, Meibergdreef 9 1105AZ, Amsterdam 1105AZ, the Netherlands. E-mail: v.b.weeda@amsterdamumc.nl

How to cite this article: Weeda VB, Murawski M. The future of pediatric hepatocellular carcinoma: a combination of surgical, locoregional, and targeted therapy. Hepatoma Res 2021;7:43. https://dx.doi.org/10.20517/2394-5079.2021.10

Received: 28 Jan 2021 First Decision: 22 Mar 2021 Revised: 30 Mar 2020 Accepted: 1 Apr 2021 Published: 11 Jun 2021

Academic Editors: Richard S Finn, Guang-Wen Cao Copy Editor: Xi-Jun Chen Production Editor: Xi-Jun Chen

\begin{abstract}
Despite hepatocellular carcinoma's position as the second most common pediatric liver tumor, it is a rare tumor in children warranting international collaboration to improve outcomes. Few cases diagnosed in earlier stages, when confined to the liver and responding to systemic treatment or with resectable metastases, may be cured by complete resection and/or orthotopic transplantation. Complete resection is the only chance for cure; therefore, all attempts should be made to make these options available. Despite modest progress in locoregional treatments, these serve in most cases as palliative treatment or as a bridge to definitive treatment at best. Currently used systemic treatments have response rates below $50 \%$. Five-year survival in advanced stages is below $30 \%$. The international Paediatric Hepatic International Tumour Trial trial is evaluating novel systemic treatments in pediatric hepatocellular carcinoma. Patients suffering from these tumors likely benefit from targeted treatment based on molecular aberrations corresponding with tumor subtype.
\end{abstract}

Keywords: Hepatocellular carcinoma, pediatric, liver tumor, liver transplantation, surgery, locoregional treatment, targeted treatment

\section{INTRODUCTION}

Despite its low incidence of approximately one per million children, hepatocellular carcinoma (HCC) is the 
second most common primary pediatric liver cancer (http://seer.cancer.gov). In adults the incidence of HCC is much higher making it the third worldwide cancer-related cause of death. HCC continues to pose a significant therapeutic challenge. Despite overall progress in pediatric and adult oncology, the cure rates in non-resectable HCC remain dismal, with 5-year overall survival ranges of $20 \%$-30\% in most multicenter trials evaluating systemic treatment. The abundance of data from both basic research efforts and clinical trials in adult HCC may be used to explore the most common aberrant molecular signaling pathways, to stratify subtypes, and to guide research efforts to learn if these findings are present in pediatric HCC as well. Stratification according to tumor subtype with accompanying molecular aberrance print may serve as a guide for targeted systemic treatment in pediatric HCC.

\section{ETIOLOGY AND CLASSIFICATION}

Pediatric HCC may be divided into "de novo" HCC and HCC developing in a setting of underlying liver disease $^{[1]}$.

De novo HCC ( 70\% of cases) may be subcategorized based on histology in three subtypes: conventional type HCC, HCC with elements of hepatoblastoma, and fibrolamellar hepatocellular carcinoma.

Conventional type HCC is histologically similar to HCC in adult patients without cirrhosis. HCC with elements of hepatoblastoma is by convention now termed hepatocellular neoplasm not otherwise specified (HCN-NOS) and may respond differently to chemotherapy based on its biology ${ }^{[2,3]}$. Fibrolamellar hepatocellular carcinoma (FL-HCC) is usually a solitary tumor, often containing a central necrotizing scar, and made up of lamellar stroma with polygonal tumor cells ${ }^{[4]}$. It predominantly occurs in older children and adolescents and comprises approximately $20 \%$ of all HCC in children ${ }^{[5]}$. It was previously thought that FLHCC had a superior prognosis to conventional HCC; however, more recent reports disprove this ${ }^{[6,7]}$.

Pediatric HCC associated with underlying disease ( $30 \%$ of cases) may originate from a number of conditions causing hepatocellular destruction. Amongst these are Alagille syndrome, alpha 1-antitrypsin deficiency, ataxia telangiectasia, auto-immune hepatitis, Fanconi anemia, Gardner syndrome, familial adenomatous polyposis, familial progressive intrahepatic cholestasis, hemochromatosis, liver mitochondrial respiratory chain disease, primary sclerosing cholangitis, transaldolase deficiency, type 1 glycogen storage disease, tyrosinemia, Wilson disease, and hepatitis B and C. The incidence of hepatitis B related pediatric HCC - which was particularly high in low- and middle-income countries in Sub-Saharan Africa, Southeast Asia, and South America - has declined significantly since the introduction of large-scale hepatitis B vaccination programs ${ }^{[8]}$.

Only a low percentage of pediatric HCC cases can be linked to cirrhosis ${ }^{[9]}$. There are indications that fewer genetic changes are needed for tumors to occur in the pediatric age group ${ }^{[10]}$. Although the end stage cirrhosis does not frequently occur in children, the process of repeated cycles of damage and repair resulting consecutively in disturbed cell signaling, hyperplasia, dysplasia, and ultimately tumor formation is highly similar to tumorigenesis in adult HCC patients ${ }^{[11]}$.

\section{MAJOR MOLECULAR ABERRANCES}

In both pediatric and adult conventional HCC, actors in growth, development, and differentiation pathways are frequently dysregulated ${ }^{[1,111]}$. Major aberrant molecular components in both pediatric and adult HCC are WNT/CTNNB1 (Beta-catenin), EPHB2 (ephrin type B receptor 2), TGFB1 (transforming growth factor beta 1), and MTOR (mechanistic target of rapamycin) ${ }^{[12]}$. 
Furthermore, in adult HCC, the angiogenesis and apoptosis pathways are frequently affected ${ }^{[11]}$. These signaling pathways are significant as the only targeted agents with some efficacy in adult HCC are sorafenib and its generational successors which are multi kinase inhibitors targeting these pathways ${ }^{[13]}$. Despite a lack of evidence that these pathways are significantly aberrant in pediatric HCC, results from a small pediatric series using sorafenib combined with cisplatin and doxorubicin have been encouraging ${ }^{[14]}$. Although results from adult HCC studies cannot be directly extrapolated to the pediatric patient group, the efficacy of sorafenib in pediatric HCC may indicate overlap and potentially similar tumor subtypes. Further development of targets and biomarkers in pediatric HCC is warranted.

\section{SURGICAL AND SYSTEMIC TREATMENT}

Complete surgical resection - including orthotopic liver transplant - is essential to cure pediatric HCC. In contrast to findings in hepatoblastoma ${ }^{[15]}$, a negative resection margin has the potential to affect long-term survival in pediatric HCC. Ziogas et al. ${ }^{[16]}$ analyzed the National Cancer Database (106 children with HCC treated between 2004 and 2015) and demonstrated the negative impact of resection with a tumor positive margin on overall survival. The difference in overall survival between patients undergoing liver transplantation and patients undergoing resection was not significantly different $(P=0.20)$. In both groups, however, increased overall survival compared to resection with a tumor positive margin was found $(P=$ 0.001 for transplantation $v s$. positive margin resection and $P=0.003$ for negative $v s$. positive margin resection). Although the difference between liver transplantation and resection with a negative margin was not significantly different, there is a clear trend toward better survival after transplantation, especially in higher tumor stages. These findings reiterate the importance of early referral for transplant evaluation. Furthermore, no difference was shown between patients who underwent liver transplant within or outside the Milan criteria ${ }^{[16]}$, once again emphasizing these criteria may not be applicable to this patient group.

However, only a small fraction of patients is eligible for surgery ( 20\%) or transplantation at diagnosis. Thus, efficacious systemic treatments are urgently needed. HCC is relatively chemoresistant with a response rate below 50\%, which does not translate into satisfactory long-term survival. Previous trials from pediatric liver tumor study groups have treated HCC with the same chemotherapy regimens as hepatoblastoma. Although there has been great progress in the outlook of high risk and advanced hepatoblastoma, efficacy in pediatric HCC is limited and there have not been significant improvements in survival due to these treatments $^{[17,18]}$.

The presently run collaborative trial on pediatric liver tumors, the Paediatric Hepatic International Tumour Trial (PHITT), has a separate treatment approach for HCC (https://clinicaltrials.gov/ct2/show/NCT03017326). In the PHITT trial, patients (younger than 30 years of age) with HCC are divided into two groups: a group of resectable HCC and a group of unresectable and/or metastatic HCC.

Patients with resectable HCC will be observed without chemotherapy after resection or transplantation if they have an underlying metabolic, genetic, or viral infection-mediated predisposing condition. The rationale behind this approach is that (1) current studies in adult HCC do not support a role of adjuvant chemotherapy, and (2) tolerance for chemotherapy in the context of possible liver dysfunction/cirrhosis is decreased. However, it is important to note that the potential role and the optimal regimen of adjuvant chemotherapy for HCC in the context of underlying disease are unknown ${ }^{[19]}$. In case of "de novo" HCC (including FL-HCC), patients will be treated with 4 cycles of cisplatin and doxorubicin ("PLADO"), as that is the only regimen pediatric tumor groups have reported some effectiveness with and no other efficacious chemotherapy is available at the moment ${ }^{[17,20]}$. 
Patients with unresectable and/or metastatic HCC will be randomized to a time-intensive treatment with PLADO with sorafenib or PLADO with sorafenib and GEMOX (gemcitabine and oxaliplatin) and reevaluated at set time points. Doxorubicin + sorafenib has been previously explored in adults with advanced HCC, and GEMOX + sorafenib is another example of a regimen that has shown some efficacy in adult HCC and is now used in pediatric patients ${ }^{[21-23]}$. A prolonged complete response in a 25 -year-old woman with FLHCC on this regimen showed potential for efficacy in a young, non-cirrhotic patient ${ }^{[24]}$. The data from the PHITT trial will need to answer whether there is efficacy in a larger group of pediatric and adolescent patients.

Identification and where possible validation of prognostic biomarkers and toxicity biomarkers will also be performed within the PHITT trial. In the meantime, analyses from molecular research efforts point in the direction of different HCC subtypes which may respond best to precision medicine ${ }^{[25-27]}$.

\section{LOCOREGIONAL TREATMENT}

When both surgery and systemic treatment fail, only locoregional treatment options remain. Transarterial chemoembolization (TACE), transarterial radioembolization with yttrium-90 (TARE-Y90), stereotactic radio frequency ablation (SRFA), and magnetic resonance imaging-guided high-intensity focused ultrasound (MR-HIFU) are such options. However, there is limited experience in children with these methods.

Recently, Aguado et al. ${ }^{[28]}$ reported a clinical trial investigating 10 patients (3 FL-HCC, 3 HCC, 2 HB, and 2 HCN-NOS) with unresectable and/or metastatic disease who received treatment with TARE-Y90 as palliative treatment or bridge to liver transplant. All patients received prior chemotherapy and/or targeted therapy. Six had laparotomies with lobectomies performed in 4 cases. One patient had TACE and RFA. Median survival post-TARE-Y90 was 4 months (range, 2-20 months). In one patient with HCN-NOS the response after TARE-Y90 allowed for a liver transplant. The possibility to provide radiation to the tumor, thus reducing toxicity, is a major advantage. TARE-Y90 is commonly used in adult patients with HCC, where it is superior to TACE in terms of time-to-progression of disease, toxicity, and posttreatment quality of life ${ }^{[22]}$.

Weiss et al ${ }^{[30]}$ described 21 TACE procedures that were performed on 8 patients with HCC (including one with FL-HCC). It was well tolerated by all 8 patients with adverse events mainly classified as grade 1 . Three patients had a partial response, five demonstrated stable disease and 2 patients died due to progression. Bridging to transplantation was achieved in 6 patients after a mean of 3 TACE procedures. The 5 -year overall survival was $83 \%$ with a mean follow up of 8.2 years (range $3.4-11$ years).

The complete response rate after SRFA is comparable with that achieved by liver resection ${ }^{[31]}$. In 2020 , Hetzer et al. ${ }^{[32]}$ presented the largest pediatric series of 10 patients with 15 liver masses (including one with HCC), who underwent SRFA. Complete radiological response was observed in all cases. Complications included temporary portal vein thrombosis, and temporary paresis of the diaphragm (each one patient). For one patient with HCC (centrally located tumor, $3 \mathrm{~cm}$ in diameter), SRFA was applied as a bridge to transplantation. Histological examination confirmed complete tumor apoptosis in this patient.

High-intensity focused ultrasound (HIFU) is a non-invasive ablation method that uses focused ultrasonic waves (0.8-1.6 MHz) to destruct the target located deep in the body (by thermic effect or by phenomenon of cavitation $)^{[33]}$. In 2013 Wang et al. ${ }^{[34]}$ presented their first experience with HIFU combined with TACE in 12 patients with unresectable hepatoblastoma. Complete ablation was achieved in 10 patients (83\%). Two 
patients died from tumor progression. The median survival time was 21.5 months, and the survival rates at 1 and 2 years were $91.7 \%$ and $83.3 \%$, respectively. There is no data on children with HCC. A clinical trial investigating feasibility and safety of MR-HIFU was reported by Napoli et al. ${ }^{[35]}$ in 2013. He reported 6 adult patients with osteoid osteoma. In 2018, Sharma et al. ${ }^{[36]}$ described their early experience with MR-HIFU ablation to treat symptomatic benign, locally aggressive, and metastatic tumors in children. Two clinical trials were designed in Children's National Medical Center to investigate feasibility and safety of MR-HIFU ablation in children with osteoid osteoma (NCT02349971, Jan 2015-Oct 2020) and with refractory or relapsed solid tumors (NCT02076906, Apr 2014-Apr 2021). HIFU seems a promising method that may be combined with systemically administered thermally sensitive chemotherapy.

\section{DISCUSSION AND CONCLUSION}

The mainstay in curative treatment of pediatric HCC is complete resection; this includes transplantation. Resection with a tumor-negative margin is crucial. In patients with pediatric HCC where there is any doubt regarding safe resection with a negative margin, early referral for transplant assessment is warranted. Milan criteria may not apply in this patient group.

Locoregional treatment modalities may serve as palliation in addition to providing options for a bridge to surgery/transplantation. Worth mentioning here are TACE, TARE-Y90, and SFRA specifically as a valuable option for small but centrally located or multifocal HCC.

Systemic treatment is indicated for patients diagnosed in advanced stages who are not primarily amenable for surgical treatment (including liver transplant) or who suffer recurrence, and adjuvant systemic treatment is indicated for patients with "de novo" HCC. The presently run PHITT trial may have the power to answer which regimens would best be used in these scenarios and results are eagerly awaited. Furthermore, ongoing molecular research efforts clarifying aberrance signatures accompanying the diverse HCC subcategories based on etiology may guide targeted treatment in the future.

\section{DECLARATIONS}

\section{Authors' contributions}

Made substantial contributions to conception and design of the study and performed data acquisition and interpretation: Weeda VB, Murawski M

\section{Availability of data and materials}

Not applicable.

\section{Financial support and sponsorship}

None.

\section{Conflicts of interest}

Both authors declared that there are no conflicts of interest.

\section{Ethical approval and consent to participate}

Not applicable.

\section{Consent for publication}

Not applicable. 


\section{Copyright}

(C) The Author(s) 2021.

\section{REFERENCES}

1. Aronson DC, Meyers RL. Malignant tumors of the liver. Sem Ped Surg 2016;25:265-75.

2. Tanaka Y, Inoue T, Horie H. International pediatric liver cancer pathological classification: current trend. Int J Clin Oncol 2013;18:946-54. DOI PubMed

3. Prokurat A, Kluge P, Kosciesza A, Perek D, Kappeler A, Zimmermann A. Transitional liver cell tumors (TLCT) in older children and adolescents: A novel group of aggressive hepatic tumors expressing beta-catenin. Med Pediatr Oncol 2002;39:510-8. DOI PubMed

4. Torbenson M. Review of the clinicopathological features of fibrolamellar carcinoma. Adv Anat Pathol 2007;14:217-23. DOI PubMed

5. Eggert T, McGlynn KA, Duffy A, Manns MP, Greten TF, Altekruse SF. Fibrolamellar hepatocellular carcinoma in the USA: 20002010: a detailed report on frequency, treatment and outcome based on the Surveillance, Epidemiology, and End Results database. Unit Europ Gastroenterol J 2013;1:351-7. DOI PubMed PMC

6. Katzenstein HM, Krailo MD, Malogolowkin MH, et al. Fibrolamellar hepatocellular carcinoma in children and adolescents. Cancer 2003;97:2006-12. DOI PubMed

7. Weeda VB, Murawski M, McCabe AJ, et al. Fibrolamellar variant of hepatocellular carcinoma does not have a better survival than conventional hepatocellular carcinoma in children: results and treatment recommendations for the Childhood Liver Tumour Strategy Group (SIOPEL) experience. Eur J Cancer 2013;49:2698-704. DOI PubMed

8. Chang M, You S, Chen C, et al; Taiwan Hepatoma Study Group. Long-term effects of hepatitis B immunization of infants in preventing liver cancer. Gastroenterol 2016;151:472-80. DOI PubMed

9. Meyers RL. Tumors of the liver in children. Surg Oncol 2007;16:195-203. DOI PubMed

10. Eichenmüller M, Trippel F, Kreuder M, Beck A, Schwarzmayr T, Haberle B, et al. The genomic landscape of hepatoblastoma and and their progenies with HCC-like features. $J$ Hepato ;61:1312-20. DOI PubMed

11. Jain S, Singhal S, Lee P, Xu R. Molecular genetics of hepatocellular neoplasia. Am J Transl Res 2010;2:105-18. PubMed PMC

12. Weeda VB, Aronson DC, Verheij J, Lamers WH. Is hepatocellular carcinoma the same disease in children and adults Comparison of histology, molecular background and treatment in pediatric and adult patients. Pediatr Blood Cancer 2019;66:e27475. DOI PubMed

13. Bruix J, Qin S, Merle P, et al; RESORCE Investigators. Regorafenib for patients with hepatocellular carcinoma who progressed on sorafenib treatment (RESORCE): a randomized, double-blind, placebo/controlled, phase 3 trial. Lancet 2017;389:56-66. DOI PubMed

14. Schmid I, Haberle B, Albert MH, et al. Sorafenib and cisplatin-doxorubicin (PLADO) in pediatric hepatocellular carcinoma. Pediatr Blood Cancer 2012;58:539-44. DOI PubMed

15. Aronson DC, Weeda VB, Maibach R, et al; Childhood Liver Tumour Strategy Group (SIOPEL). Microscopically positive resection margin after hepatoblastoma resection: what is the impact on prognosis? Eur J Cancer 2019;106:126-32. DOI PubMed

16. Ziogas IA. Benedetti DJ, Matsuoka LK, et al. Surgical management of pediatric hepatocellular carcinoma: An analysis of the National Cancer Database. J Pediatr Surg 2020;S0022-3468(20)30433-4. DOI PubMed

17. Czauderna P, Mackinlay G, Perilongo G, et al; Liver Tumors Study Group of the International Society of Pediatric Oncology. Hepatocellular carcinoma in children: Results of the first prospective study of the International Society of Pediatric Oncology group. $J$ Clin Oncol 2002;20:2798-804. DOI PubMed

18. Murawski M, Weeda VB, Maibach RM, Morland B, Roebuck D, Zimmerman A, et al. Hepatocellular Carcinoma in Children: Does modified platinum- and doxorubicin-based chemotherapy increase tumor resectability and change outcome? J Clin Oncol 2016;34:1050-6. DOI PubMed

19. Schmid I, von Schweinitz D. Pediatric hepatocellular carcinoma: challenges and solutions. J Hepatocell Carcinoma 2017;4:15-21. DOI PubMed PMC

20. Katzenstein HM, Krailo MD, Malogolowkin MH, et al. Hepatocellular Carcinoma in Children and Adolescents: Results from the Pediatric Oncology Group and the Children's Cancer Group Intergroup Study. J Clin Oncol 2002;20:2789-97. DOI PubMed

21. Richly H, Schultheis B, Adamietz IA, et al. Combination of sorafenib and doxorubicin in patients with advanced hepatocellular carcinoma: Results from a phase I extension trial. Eur J Cancer 2009;45:579-87. DOI PubMed

22. Liu Y, Yue H, Xu S, et al. First-line gemcitabine and oxaliplatin (GEMOX) plus sorafenib, followed by sorafenib as maintenance therapy, for patients with advanced hepatocellular carcinoma: a preliminary study. Int J Clin Oncol 2015;20:952-9. DOI PubMed

23. Assenat E, Pageaux GP, Thézenas S, et al. Sorafenib alone vs. sorafenib plus GEMOX as $1^{\text {st }}$-line treatment for advanced HCC: the phase II randomized PRODIGE 10 trial. Br J Cancer 2019;120:896-902. DOI PubMed PMC

24. Gras P, Truant S, Boige V, et al. Prolonged complete response after GEMOX chemotherapy in a patient with advanced fibrolamellar hepatocellular carcinoma. Case Rep Oncol 2012;5:69-72. DOI PubMed PMC

25. Haines K, Sarabia SF, Alvarez KR, et al. Characterization of pediatric hepatocellular carcinoma reveals genomic heterogeneity and diverse signaling pathway activation. Pediatr Blood Cancer 2019;66:e27745. DOI PubMed

26. Cho SJ. Pediatric liver tumors: Updates in classification. Surg Pathol Clin 2020;13:601-23. DOI PubMed

27. Ranganathan S, Lopez-Terrada D, Alaggio R. Hepatoblastoma and pediatric hepatocellular carcinoma: An update. Pediatr Dev Pathol 2020;23:79-95. DOI PubMed

28. Aguado A, Ristagno R, Towbin AJ, et al. Transarterial radioembolization with yttrium-90 of unresectable primary hepatic malignancy in children. Pediatr Blood Cancer 2019;66:e27510. DOI PubMed 
29. Kallini JR, Gabr A, Salem R, Lewandowski RJ. Transarterial radioembolization with yttrium-90 for the treatment of hepatocellular carcinoma. Adv Ther 2016;33:699-714. DOI PubMed PMC

30. Weiss KE, Sze DY, Rangaswami AA, et al. Transarterial chemoembolization in children to treat unresectable hepatocellular carcinoma. Pediatr Transplant 2018;22:e13187. DOI PubMed

31. Pompili M, Saviano A, de Matthaeis N, et al. Long-term effectiveness of resection and radiofrequency ablation for single hepatocellular carcinoma $</=3 \mathrm{~cm}$. Results of a multicenter Italian survey. $J$ Hepatol 2013;59:89-97. DOI PubMed

32. Hetzer B, Vogel GF, Entenmann A, et al. Stereotactic radiofrequency ablation of a variety of liver masses in children. Int $J$ Hypertherm 2020;37:1074-81. DOI PubMed

33. Diana M, Schiraldi L, Liu Y-Y, et al. High intensity focused ultrasound (HIFU) applied to hepato-biliopancreatic and the digestive system-current state of the art and future perspectives. HepatoBiliary Surg Nutr 2016;5:329-44. DOI PubMed PMC

34. Wang S, Yang C, Zhang J, et al. First experience of high-intensity focused ultrasound combined with transcatheter arterial embolization as local control for hepatoblastoma. Hepatology 2014;59:170-7. DOI PubMed

35. Napoli A, Mastantuono M, Cavallo Marincola B, et al. Osteoid osteoma: MRguided focused ultrasound for entirely noninvasive treatment. Radiology 2013;267:514-21. DOI PubMed

36. Sharma KV, Yarmolenko PS, Eranki A, Partanen A, Celik H, Kim A. Magnetic Resonance Imaging-guided High-intensity Focused Ultrasound Applications in Pediatrics: Early Experience at Children's National Medical Center. Top Magn Reson Imaging 2018;27:4551. DOI PubMed 